University of Wisconsin Milwaukee

UWM Digital Commons

School of Information Studies Faculty Articles

Information Studies (School of)

Fall 10-2012

\title{
Measuring Author Research Relatedness: A Comparison of Word-based,Topic-based and Author Cocitation Approaches
}

Kun Lu

University of Oklahoma Norman Campus, kunlu@ou.edu

Dietmar Wolfram

University of Wisconsin - Milwaukee School of Information Studies, dwolfram@uwm.edu

Follow this and additional works at: https://dc.uwm.edu/sois_facpubs

Part of the Scholarly Communication Commons

\section{Recommended Citation}

Lu, Kun and Wolfram, Dietmar, "Measuring Author Research Relatedness: A Comparison of Word-based,Topic-based and Author Cocitation Approaches" (2012). School of Information Studies Faculty Articles. 1.

https://dc.uwm.edu/sois_facpubs/1

This Article is brought to you for free and open access by UWM Digital Commons. It has been accepted for inclusion in School of Information Studies

Faculty Articles by an authorized administrator of UWM Digital Commons. For more information, please contact open-access@uwm.edu. 
This is the accepted version of the following article: Lu, K., \& Wolfram, D. (2012). Measuring author research relatedness: A comparison of word-based, topic-based, and author cocitation approaches. Journal of the American Society for Information Science and Technology, 63(10), 1973-1986. DOI: 10.1002/asi.22628

which has been published in final form at: http://onlinelibrary.wiley.com/doi/10.1002/asi.22628/full.

\title{
Measuring Author Research Relatedness: A Comparison of Word-based, Topic-based and Author Cocitation Approaches
}

\author{
Kun Lu \& Dietmar Wolfram \\ School of Information Studies, University of Wisconsin-Milwaukee \\ P.O. Box 413, Milwaukee, WI 53201 \\ E-mail: kunlu@ou.edu, dwolfram@uwm.edu
}

Keywords: Author relatedness; Science maps; Multidimensional scaling; Topic model; Co-word analysis

\begin{abstract}
Relationships between authors based on characteristics of published literature have been studied for decades. Author cocitation analysis using mapping techniques has been most frequently used to study how closely two authors are thought to be in intellectual space based on how members of the research community co-cite their works. Other approaches exist to study author relatedness based more directly on the text of their published works. In this study we present static and dynamic word-based approaches using vector space modeling, as well as a topic-based approach based on Latent Dirichlet Allocation for mapping author research relatedness. Vector space modeling is used to define an author space consisting of works by a given author. Outcomes for the two word-based approaches and a topic-based approach for 50 prolific authors in library and information science are compared with more traditional author cocitation analysis using multidimensional scaling and hierarchical cluster analysis. The two word-based approaches produced similar outcomes except where two authors were frequent co-authors for the majority of their articles. The topic-based approach produced the most distinctive map.
\end{abstract}




\section{Introduction}

The study of scientific production measured through publications has a long history. To better understand patterns and relationships in scientific production, various tools have been developed. Science mapping is one of the most useful tools to visualize scientific structure. It helps to identify scientific themes, and discover new knowledge. The unit of interest for mapping may include authors, articles, and journals. The essence of a science map is the measure of relatedness among the units. To date, five approaches have been used to measure the relatedness between authors, where the nature of the relationship studied is based on the data used: direct citation, cocitation analysis, co-authorship analysis, bibliographic coupling analysis and co-word analysis (discussed below). All have been successfully applied to visualize scientific

structure and to describe author relatedness. Recently, more sophisticated hybrid methods (i.e. using textual content and citations) have been applied to the mapping of articles (Cao \& Gao, 2005; Ahlgren \& Colliander, 2009; Boyack \& Klavans, 2010) and journals (Liu et al., 2010). To the best of our knowledge the uses of textual content and, more specifically, a topic model (e.g. Deerwester et al., 1990) to determine the relatedness of authors have not been studied yet.

In this study we propose new textual feature-based approaches based on co-occurring words that apply vector space modeling to measure the relatedness of authors' research. A topic-based approach using Latent Dirichlet Allocation (LDA) modeling is also applied to capture the latent topical features from the occurrence and the co-occurrence of words within a document and across documents created by authors. Two authors will be similar to each other if they write similar content and topics. These new approaches can be used as complementary techniques to those currently used to generate author maps.

More specifically, the purpose of the present research is to:

1. Propose three new methods, two word-based, one topic-based, to measure author research relatedness based on the content of their publications. 
2. Compare multidimensional scaling (MDS) and hierarchical clustering outcomes of the proposed wordbased models, the topic-based model and the widely used author cocitation analysis (ACA) for a group of authors.

As an initial investigation of these topics, our focus will be on authors whose publications appear in the highest impact library and information science journals.

\section{Related Work}

The literature review section covers two parts. The first section reviews existing techniques used for mapping bibliometric units. The second section briefly reviews the relevant models used in the study. It includes an introduction to the essential ideas of the vector space model, how it applies to the current study, and provides a short introduction to the LDA or topic model.

\section{Bibliometric Relatedness Measures}

Many bibliometric studies have formulated quantitative measures to map scientific structure at different levels of granularity including authors, articles and journals. In reviewing visualization studies for knowledge domains, Börner, Chen and Boyack (2005) categorized relatedness measures into two broad categories: citation linkages and co-occurrence similarities. Within the relatedness measures, five basic approaches were identified: direct citation, cocitation analysis, co-authorship analysis, bibliographic coupling and co-word analysis.

\section{Direct citation}

Direct citation accounts for the relatedness between a citing work and a cited work based on citing behavior. This measure is usually asymmetric. Shibata et al. (2008) explored citation networks for two research domains and divided the networks into clusters in order to identify research fronts. Direct citation has not attracted wide attention. One possible reason may be its requirement for a very long time window to obtain a sufficient linking signal for clustering (Boyack \& Klavans, 2010). 


\section{Bibliographic coupling}

The idea that two articles that share the same references are related, referred to as bibliographic coupling, was outlined by Kessler (1963). The more references two articles have in common, the more closely related they are thought to be. Note that this list is static over time because references within articles do not change. With the interrelation of this link, scientific products can be ordered into groups. Weinberg (1974) reviewed the theory and practical applications of bibliographic coupling and granted the usefulness of the method. More recently, Zhao and Strotmann (2008) aggregated bibliographic coupling at an author's oeuvre (body of work) level, which they called Author Bibliographic-Coupling Analysis (ABCA). They found ABCA can provide an effective picture of current active research in a field.

\section{Cocitation analysis}

Cocitation analysis, introduced by Small (1973), is probably the most influential approach for assessing relatedness measures. If two articles are cited by the same third article, these two articles are co-cited. The assumption is that the appearance of two articles in the same reference list indicates a semantic association between the articles. Unlike traditional bibliographic coupling, cocitation is a dynamic relationship based on the citing authors. New citing authors can change the cocitation relationship. This feature is important because science is developing continuously. Relationships among scientific units being studied should be able to incorporate this dynamic change.

White and Griffith (1981) first applied cocitation techniques to authors, called author cocitation analysis or ACA. The essential transformation is to consider "Author" as a body of writings by a person (i.e. an oeuvre). So the cocitation of authors applies to any work by any author being co-cited with any work by another author. Multidimensional scaling and factor analysis have been employed to describe the scientific structure of information science authors. Since then, a number of studies have been conducted using variations of the ACA method, including normalization (Ahlgren, Jarneving \& Rousseau, 2003; White, 2003; Leydesdorff \& Vaughan, 2006; van Eck \& Waltman, 2009), author counts (Zhao \& 
Strotmann, 2011) and last author ACA (Zhao \& Strotmann, 2010). One disadvantage of cocitation analysis is the lack of cognitive interpretation of the relatedness of the co-cited units. Without enough domain knowledge, one can hardly interpret the cocitation map. Leydesdorff (1987) argued that cocitation maps only partially represent the structure of science. One possible solution to this problem is to interpret the ACA map with word analysis. Toward this end, Braam et al. (1991) combined cocitation and word analysis. Word-profile analysis was used to examine the cognitive relatedness of documents within the same cocitation cluster.

\section{Co-authorship analysis}

A co-authorship relationship is established when authors co-publish a paper. Glänzel (2001) studied international co-authorship links to reveal the structures in international collaborations. Liu et al. (2005) constructed a network with co-authorship relations in the field of digital libraries. Ding (2011b) studied scientific collaborations and citation patterns of researchers and combined the results with a topic model approach to examine collaborations among researchers who share similar and different research interests.

Although co-authorship has been considered one measure of author relatedness, it reflects a stronger social tie among the collaborating authors than any other relatedness measure. It is this feature of coauthorship that makes co-authorship analysis more revealing of a social network rather than a scientific structure.

\section{Co-word analysis}

Co-word analysis collects evidence of relatedness from co-occurring keywords from different articles. Compared with the approaches introduced earlier, co-word analysis directly uses actual contents to measure relatedness whereas the others find indirect evidence through citation and co-author relations. An obvious advantage of co-word analysis is that relatedness can be interpreted directly according to document contents. 
Coulter et al. (1998) mapped the discipline of software engineering with co-word analysis. Indexing terms from the ACM computing Classification System were used as the unit of analysis. Ding et al. (2001) conducted a co-word analysis on a sample of 2,012 articles from the Web of Science (WoS) to reveal themes of information retrieval research. Both professionally assigned keywords and keywords from titles and abstracts were extracted. Standardization was applied to map the keywords to a controlled vocabulary. The study demonstrated the feasibility of co-word analysis as a method to extract patterns from a text corpus. In these co-word analysis works, the co-occurrences of keywords in articles were used as an indication of their association strengths to map the relatedness of the keywords. In the present study, words will be used to determine higher level relatedness: the relatedness of authors. Two authors are similar to each other if they have written similar content.

Like other approaches, co-word analysis has its own weaknesses. Leydesdorff (1997) noted that the meaning of words change from position to position and from one text to another. He also suggested this change will destabilize the science map produced by co-word analysis. Another disadvantage of using indexer assigned keywords as the source for co-word analysis is the "indexer effect" (Law \& Whittaker, 1992), which creates bias through factors such as the artificiality of an indexing language, delays in changes to the indexing language to reflect the current state of a discipline, and subjectivity in the assignment of index terms.

\section{Background Information on Relevant Models to be Used}

In the current study, the vector space model, applied widely in information retrieval research, serves as the framework to determine author relatedness for two word-based approaches. LDA topic modeling is used to determine author relatedness for the topic-based approach. The following sections provide a brief review of the relevant models. 


\section{Vector Space Modeling}

The vector space model is one of the most influential models in information retrieval (Salton and McGill, 1983). In this model, each document is represented as a vector and the elements of the vector consist of words appearing in the collection. The document vectors in a collection constitute a document term matrix. The value of each element represents the term significance in the document. By virtue of the vector space model, documents are transformed into vectors. Traditional measures like angle (e.g. based on a cosine measure) and distance (e.g. Euclidean distance) can be used to measure the similarity between documents. In the vector space, a number of documents constitute a document space. The centroid of the document space is a summarization of the characteristics of the space. It represents the average vector for a group of documents.

In the current study, all of the articles in our data collection will constitute a collection space. Each author will be viewed as a document space consisting of the articles he/she has written. This space is a subspace of the collection space, named the author space. The centroid of the author space will be used to represent the author. The relatedness between authors will be measured through the similarity between the centroids of their author spaces.

\section{Topic Model- Latent Dirichlet Allocation}

The vector space model assumes independence among the words in the documents. However, in the real world, this assumption is rarely valid because the terms are associated with each other due to their semantics. The topic model is an improvement over the basic vector space model in terms of relieving the independence assumption and capturing the term associations. Instead of assuming independence among terms, the topic model assumes exchangeability among terms in documents, which is a much looser assumption. Early works on the topic model include Latent Semantic Indexing (LSI) by Deerwester et al. (1990) and the probabilistic LSI (pLSI) by Hofmann (1999). LDA is a more recent technique proposed by 
Blei et al. (2003). It has an advantage over LSI in explicitly modeling the latent topics, and over pLSI in solving the over-fitting problem (i.e. a model with too many parameters).

The LDA model treats a document as a mixture of topics and a topic as a mixture of terms. Each document (i.e. a mixture of topics $\theta$ ) is generated from a latent Dirichlet distribution with a prior of $\alpha$, and each topic (i.e. a mixture of terms $\varphi_{k}$ ) is generated from a Dirichlet distribution with a prior of $\beta$. The generation process entails first, sampling a document $\theta_{d}$ from $\operatorname{Dir}(\alpha)$. At each position of a word in a document, a topic $z$ is selected according to $\theta_{d}$, and a word $w$ is selected according to $z$ and $\varphi_{k}$. Figure 1 plots the plate notation of the generating process:

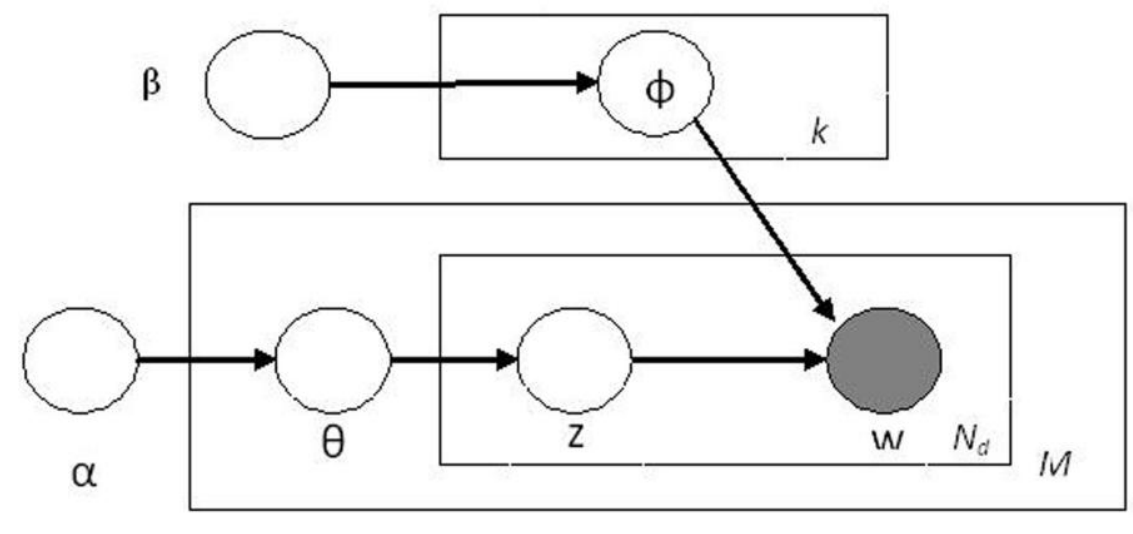

FIG. 1. Graphic model representation of LDA.

In the above figure, white circles indicate latent variables and gray circles indicate observed variables. Arrows indicate conditional dependencies between variables. Plates indicate repeated sampling and the number in the lower right of the plate indicates the number of repetitions. So $k$ is the number of topics, $N_{d}$ is the length of a document, and $M$ is the number of documents in the collection. In the model, $\alpha$ and $\beta$ are hyperparameters that define the nature of the priors on $\theta$ and $\varphi$.

Rosen-Zvi et al. (2010) extended the original LDA model to include authors and proposed the authortopic model (Figure 2). 


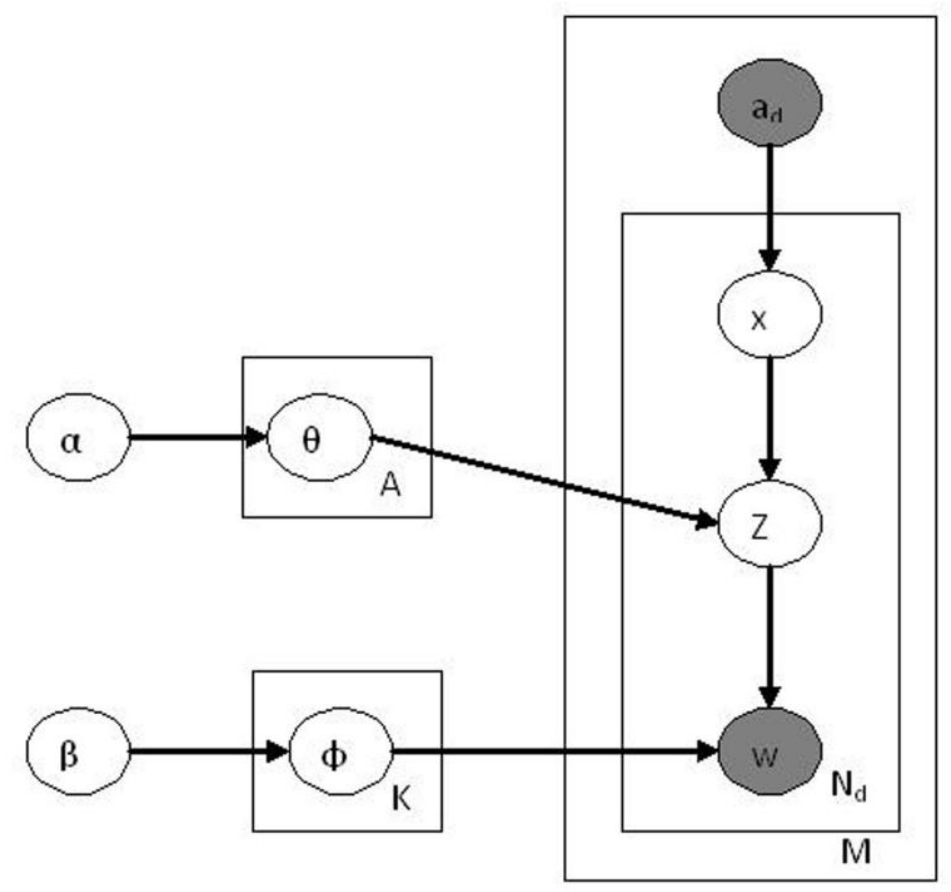

FIG. 2. Graphic model representation of author-topic model.

This model includes authorship information in the generative process. Each document has a number of authors $a_{d}$. Each author is considered as a distribution of topics drawn from a Dirichlet distribution with a prior of $\alpha$. For each word in a document, an author $x$ is randomly drawn from $a_{d}$ and the topic distribution associated with this author is $\theta_{x}$. Then a topic $z$ is selected the same way as in a LDA model to generate the observed word $w$. The advantage of this author-topic model is that it adds authorship information to the model, so that the topics are learned and assigned to documents accordingly. In the output of this model, each author is a distribution of different topics; each topic is a distribution of terms. As the purpose of the current study is to measure the relatedness of authors, the author-topic model will be appropriate to produce author similarities based on their topics. Gibbs sampling (Griffiths \& Steyvers, 2004) is used to estimate the parameters in the model. 


\section{Method}

\section{Data Collection}

Journals with the highest impact factor in the category of "information science \& library science" (LIS) appearing in the Journal Citation Report 2009 Social Sciences Edition were identified. Journals associated with allied subject areas such as Management Information Systems and Medical Informatics, were excluded. Table 1 lists the eight journals selected for inclusion in the study. Although ARIST (Annual Review of Information Science and Technology) publishes reviews and not research articles, these publications still represent topical areas of expertise of the authors. Bibliographic records for documents published in these journals between 2000 and 2010 were downloaded. Records downloaded were further limited to three document types: articles, proceedings papers and reviews. The other document types were less likely to represent research contributions by the authors.

TABLE 1. Selected journals.

\begin{tabular}{|l|l|l|l|}
\hline Journal Title & $\begin{array}{l}\text { Impact } \\
\text { Factor }\end{array}$ & $\begin{array}{l}\text { \# of records retrieved } \\
\text { before refinement } \\
\text { document types }\end{array}$ & $\begin{array}{l}\text { \# of records } \\
\text { downloaded }\end{array}$ \\
\hline Journal of Informetrics & 3.379 & 172 & 162 \\
\hline $\begin{array}{l}\text { Annual Review of Information Science and } \\
\text { Technology }\end{array}$ & 2.929 & 135 & 118 \\
\hline $\begin{array}{l}\text { Journal of the American Society for Information } \\
\text { Science and Technology (covering the years 2001- } \\
\text { 2010) }\end{array}$ & 2.3 & 1897 & 1451 \\
\hline Scientometrics & 2.167 & 1485 & 1390 \\
\hline Information Processing \& Management & 1.783 & 881 & 749 \\
\hline Journal of Information Science & 1.706 & 548 & 495 \\
\hline Online Information Review & 1.423 & 1053 & 482 \\
\hline Journal of Documentation & 1.405 & 844 & 380 \\
\hline Total & & 7015 & 5227 \\
\hline
\end{tabular}

In total, 5,227 records were downloaded from WoS. The raw WoS records were processed, and only three fields were kept: the article title (i.e. "TI” field), the Keywords Plus (i.e. "ID” field), and the abstract (i.e. 
“AB” field). The records then were indexed with the widely used Lemur information retrieval toolkit (http://www.lemurproject.org/). Stop words were removed and stemming was applied.

\section{Author Selection}

From the 5,227 records downloaded, we were able to identify 6,282 different author names using string matching. Because it is impractical to map all of the authors in our collection, we selected the 50 most prolific authors according to the WoS "analyze results" function. A larger number of authors could be selected, but would result in a more densely populated map that would be more difficult to interpret when all of the names are superimposed. We selected the most prolific authors because the more an author writes, the better the algorithm used "understands" her/his interests, and thus the more accurate our assessment will be.

\section{Author Space}

With the 5,227 records, we identified the authorship relation between each of the top authors using the articles they wrote. For each author in our author list we then generated an author space which consists of all the articles he/she wrote. TF*IDF term weighting was employed to assign term significance in the space. Terms that were single characters or only consisted of digits (e.g. "2001”) were filtered out. We believe that these terms add noise into the space rather than meaning. The relatedness between authors is measured through the cosine between the centroids of the author spaces.

\section{Static author space vs. dynamic author space}

Using the content of publications to determine the strength of the relationship between authors introduces a potentially confounding factor. The similarity between co-authors may be high because the text of the publications they have co-written will be used to determine the strength of their relationships. One could argue that this creates a biased assessment of the strength of the relationship because there is an exact match for the text of the co-authored publications that creates a stronger bond than for two authors who have published in a common area but did not collaborate. On the other hand, the simple fact that the 
collaboration has resulted in one or more co-authored documents should be acknowledged as a strong tie between the authors. The strength of the relationship can be assessed both ways, where the common publications are included or excluded. Accordingly, we propose a static author space and dynamic author space to fulfill this goal. In a static space, each author has her/his own space which consists of her/his articles. This space does not change when measuring author relatedness. In the static author space, the coauthorship is not controlled. The relatedness of authors will include the similarity arising from the strength of the co-authorships. Conversely, in the dynamic author space, the author spaces depend on a pair of authors. Co-authored articles by the pair of authors are excluded. In this case, each author may have a different author space when measured with different authors. Within the dynamic space the coauthorship similarity is controlled because the co-authored articles are filtered out before the similarity is calculated. It is of interest to understand how two authors are related with or without their collaborative works.

\section{Measure of Relatedness}

The vector space model provides a number of readily available measures of relatedness. The most popular is the cosine measure, which measures the cosine of the angle formed by two vectors in the space. It basically measures the term weight distribution between two vectors. The more similar the distribution is, the higher the cosine value is expected to be. Therefore, the cosine similarity measure is adopted in our study to measure the relatedness between the centroids of two author spaces.

\section{Topic Model Training}

Gibbs sampling (Griffiths \& Steyvers, 2004) was used to estimate the parameters in the author-topic model. We set the number of iterations to 1,000 . The hyperparameter $\alpha$ was set to $50 / K$ where $K$ is the number of topics and hyper $\beta$ is set to 0.01 . We tested different $K$, or numbers of topic, values and decided to report the results from $K=20$ because it produced the most reasonable outcome by our judgment. Too few topics do not allow authors to be distinguished, whereas too many may cause 
relationships to be weaker. Perplexity analysis could have been applied to decide the number of topics (Blei, Ng \& Jordan, 2003). However, perplexity measures the generalization ability of a trained model which does not have much meaningful interpretation in our study because we are interested in identifying author relatedness based on our data. The topic model toolbox was employed to perform the learning process (http://psiexp.ss.uci.edu/research/programs_data/toolbox.htm). The result from the topic model training is provided in the appendix. The table includes the top words and authors for each topic as well as their probabilities.

\section{Mapping and Comparison with Cocitation Outcome}

An author-topic LDA model (Rosen-Zvi et al., 2010) was trained on our collection and a pair-wise cosine similarity measure comparison of the 50 authors was conducted, resulting in a symmetric matrix of similarity values based on the LDA modeling. Similarity matrices were also calculated for both the static and dynamic author spaces. Multidimensional scaling was used to visualize the relationships among the authors. A more traditional cocitation matrix for the 50 authors was also generated to permit a subjective comparison between the static and dynamic word-based, LDA, and author cocitation outcomes. The cocitation counts were extracted from the cited reference (i.e. "CR" field) of our data, so only the first author cocitation was tallied. Because the data represent a type of similarity measure, SPSS PROXSCAL was used to construct the map, as recommended by Leydesdorff and Vaughan (2006). To provide additional insights into the grouping of the authors, hierarchical cluster analysis (complete linkage method) was used in SPSS to superimpose groups of authors on the MDS maps to provide an additional means to assess the coherence in the resulting proximities between authors. MDS map outcomes could also be interpreted without these generated clusters. 


\section{Results}

\section{Basic Collection Statistics}

Table 2 includes some basic statistics for the collection we used to generate the author maps. After tokenization of the field contents 916,383 tokens, or individual words, were identified; the number of unique tokens, or distinct words, was 12,537. The average document length was 175.32 tokens.

TABLE 2. Basic collection statistics.

\begin{tabular}{|c|c|c|c|c|}
\hline \# of authors & \# of documents & \# of tokens & \# of unique tokens & Avg. doc length \\
\hline 6228 & 5227 & 916,383 & 12,537 & 175.32 \\
\hline
\end{tabular}

\section{Author Similarities}

An obvious advantage of the text-based similarity is that the link between authors is interpretable. When we computed pair-wise similarities for the authors, we also calculated the top contributing terms (or topics for the LDA) so that we could better understand the reason why two authors are similar. To provide a sense of how these top terms help us to understand the links, we list a number of author similarities from the static author map in Table 3. The outcome for the dynamic map has the same format. For the LDA map, the terms are replaced with topics.

According to Table 3 "Thelwall, M" and "Glanzel, W" have a similarity of 0.39 in the static map, in which "citation" contributes the $12 \%$ of the similarity, "link" $6 \%$, followed by "impact" $4 \%$, "science" $3 \%$ and "subject" $2 \%$. With the information provided by the top terms, one can see how the two authors are related. To help read the similarity values, basic descriptive statistics of the values of the pair-wise similarity for static author map, dynamic author map and LDA map are provided in Table 4. For the top 50 authors, there are 1,225 pairs of similarity values in total for each map. 
TABLE 3. A demonstration of author similarities and the top contributing terms.

\begin{tabular}{|c|c|c|c|}
\hline Author 1 & Author 2 & Similarity & Top contributing terms \\
\hline THELWALL_M & GLANZEL_W & 0.39 & $\begin{array}{l}\text { citat: } 12 \% \text { link:6\% impact:4\% scienc:3\% } \\
\text { subject:2\% }\end{array}$ \\
\hline SPINK_A & JANSEN_BJ & 0.83 & $\begin{array}{l}\text { search:26\% queri: } 11 \% \text { web:10\% engin:9\% } \\
\text { session:5\% }\end{array}$ \\
\hline BAR-ILAN_J & WOLFRAM_D & 0.40 & page: $14 \%$ search:11\% web:8\% tag:5\% engin: $4 \%$ \\
\hline EGGHE_L & BURRELL_QL & 0.47 & $\begin{array}{l}\text { informetr: } 12 \% \text { distribut:7\% index:7\% curv:5\% } \\
\text { concentr: } 4 \%\end{array}$ \\
\hline CHEN_HC & YANG_CC & 0.39 & $\begin{array}{l}\text { chines: } 8 \% \text { web:6\% search:6\% english:5\% } \\
\text { user: } 4 \%\end{array}$ \\
\hline NICHOLAS_D & HUNTINGTON_P & 0.99 & $\begin{array}{l}\text { log:14\% kiosk:5\% behaviour:5\% site:4\% } \\
\text { health: } 4 \%\end{array}$ \\
\hline BORNMANN_L & DANIEL_HD & 0.97 & $\begin{array}{l}\text { fellowship:8\% manuscript:7\% review:7\% } \\
\text { reject:5\% peer:5\% }\end{array}$ \\
\hline JANSEN_BJ & BURRELL_QL & 0.08 & $\begin{array}{l}\text { model:5\% time: } 4 \% \text { process:3\% investig:3\% } \\
\text { approxim:3\% }\end{array}$ \\
\hline WOLFRAM_D & VAKKARI_P & 0.29 & search:17\% queri:7\% term:6\% session:5\% ir:3\% \\
\hline GLANZEL_W & MOED_HF & 0.55 & $\begin{array}{l}\text { citat:15\% journal:12\% impact:6\% bibliometr:5\% } \\
\text { indic:3\% }\end{array}$ \\
\hline
\end{tabular}

TABLE 4. Descriptive statistics of similarity values in three maps $(\mathrm{N}=1225)$.

\begin{tabular}{|c|c|c|c|c|c|}
\hline & Mean & Median & Std. Deviation & Minimum & Maximum \\
\hline Static author map & 0.2352 & 0.2100 & 0.1122 & 0.05 & 0.99 \\
\hline Dynamic author map & 0.2276 & 0.2100 & 0.0949 & 0.00 & 0.65 \\
& & & & & 0.03 \\
\hline LDA map & 0.4106 & 0.3500 & 0.2430 & & 0.97 \\
\hline
\end{tabular}


The average similarity value for the LDA map is much higher than the two word-based maps. This may be due to the effect of the topic model. As a topic consists of a mixture of terms, to measure topical similarity it is possible that two different terms (e.g. "car" and "vehicle") will be considered topically similar and will then contribute to the similarity. This is helpful for identifying relatedness arising from mismatched terminology.

\section{Map Comparison}

Four author similarity maps were generated: a static author map, a dynamic author map, a LDA author map, and an author cocitation map. The static author map and dynamic author map were constructed from the similarities between the author spaces. The difference is that the former map includes the similarities for co-authored works, whereas the latter excludes these similarities when calculating the pair-wise similarity. The LDA map is built on topical similarity. The cocitation map serves as a comparison here. An examination of the pair-wise correlation of these author relatedness measures reveals significant and moderate level correlations between the word-based, topicbased and author cocitation measures (Table 5). It is not surprising that the static author map has a high correlation with the dynamic author map (Kendall's tau $b=0.971$ ). Similarly, the correlations among the three content-based approaches are generally higher than their correlations with the cocitation approach. This provides preliminary evidence that they measure different types of relationships.

Outcomes from the hierarchical cluster analysis were superimposed on the maps. Labels for the author groups were assigned by us according to the themes inherent in the top-weighted terms in each cluster. The number of clusters selected was based on the joining distance at which clusters were combined in the cluster dendrogram. A large distance between clusters before being joined provides an indication of the distinctiveness of the clusters. Two to four clusters are displayed on each map based on a large clustering distance. The same number of clusters could have been selected for all the maps, but the linking distances between some agglomerations was so small that the groups would not have been as distinctive. In all cases, the largest singular group consists of authors who work with different aspects of metrics-based 
studies, which is labeled as "Informetrics" in general in the two word-based maps and "Scientific impact evaluation" in the other two maps. This labeling indicates that the metrics-related topics have been a frequently investigated theme by the prolific authors in the selected journals during the first decade of twenty-first century. It is also noteworthy that the topic groupings of each of the maps largely aligns along the horizontal or vertical axis, with one side representing information retrieval (system and behavior) and web studies, with the other side corresponding to metrics-based or scientific evaluation studies.

TABLE 5. Correlations between Different Measures ( $\mathrm{N}=1225)$.

\begin{tabular}{|l|l|l|l|l|}
\hline & Static Author Map & Dynamic Author Map & LDA & $\begin{array}{l}\text { Author Cocitation } \\
\text { Map }\end{array}$ \\
\hline Static Author Map & 1.00 & $0.971^{* *}$ & $0.487^{* *}$ & $0.433^{* *}$ \\
\hline Dynamic Author Map & $0.971^{* *}$ & 1.00 & $0.476^{* *}$ & $0.432^{* *}$ \\
\hline LDA & $0.487^{* *}$ & $0.476^{* *}$ & 1.00 & $0.401^{* *}$ \\
\hline $\begin{array}{l}\text { Author Cocitation } \\
\text { Map }\end{array}$ & $0.433^{* *}$ & $0.432^{* *}$ & $0.401^{* *}$ & 1.00 \\
\hline
\end{tabular}

(Values in cells are Kendall's tau-b correlation, ** indicates significant at the 0.01 level with 2-tailed test)

\section{Static author map and dynamic author map comparison}

As is shown from the maps, the static map (Figure 3) and dynamic map (Figure 4) are generally consistent in terms of the location of the authors which indicates that the exclusion of similarities resulting from collaborations does not affect the overall layout. However, drastic changes may happen to individuals who have collaborated frequently with another author. One notable change is for "Bornmann_L" and "Daniel_HD" who were co-authors in a large portion of their works included in this study. They have a similarity of 0.97 in the static map. After removing the collaborative works their similarity becomes 0.12 which indicates that their non-collaborative works are not as similar. In the case of "Jansen_BJ" and "Spink_A", who have collaborated frequently with each other and have each written in similar areas separately, they are close to each other in both maps. This indicates that they are similar 
to each other irrespective of whether collaborative works are included or not. In an extreme case where "Huntington_P" co-authored with "Nicholas_D" on every article in our data collection, we treated their similarity as a missing value in the dynamic map. Both the static map and dynamic map have the most distinctive cluster separations when the hierarchical clustering is viewed from the two cluster level. In both maps, the left half consists of researchers from information retrieval and the right half represents the authors who contributed to the informetrics area. Several authors are close to the cluster boundary and one switches between the two clusters ("Ding_Y"), indicating that their research interests could overlap both areas. Given the high correlation between the static and dynamic author maps, it is expected that the overall layouts will be very similar in both maps. This observation may not be generalized to other data collections. Which map to explore depends on whether one wishes to have the co-authorship relation embedded or not. However, it should be noted that the static author map is more computationally efficient than the dynamic author map because less processing is needed.

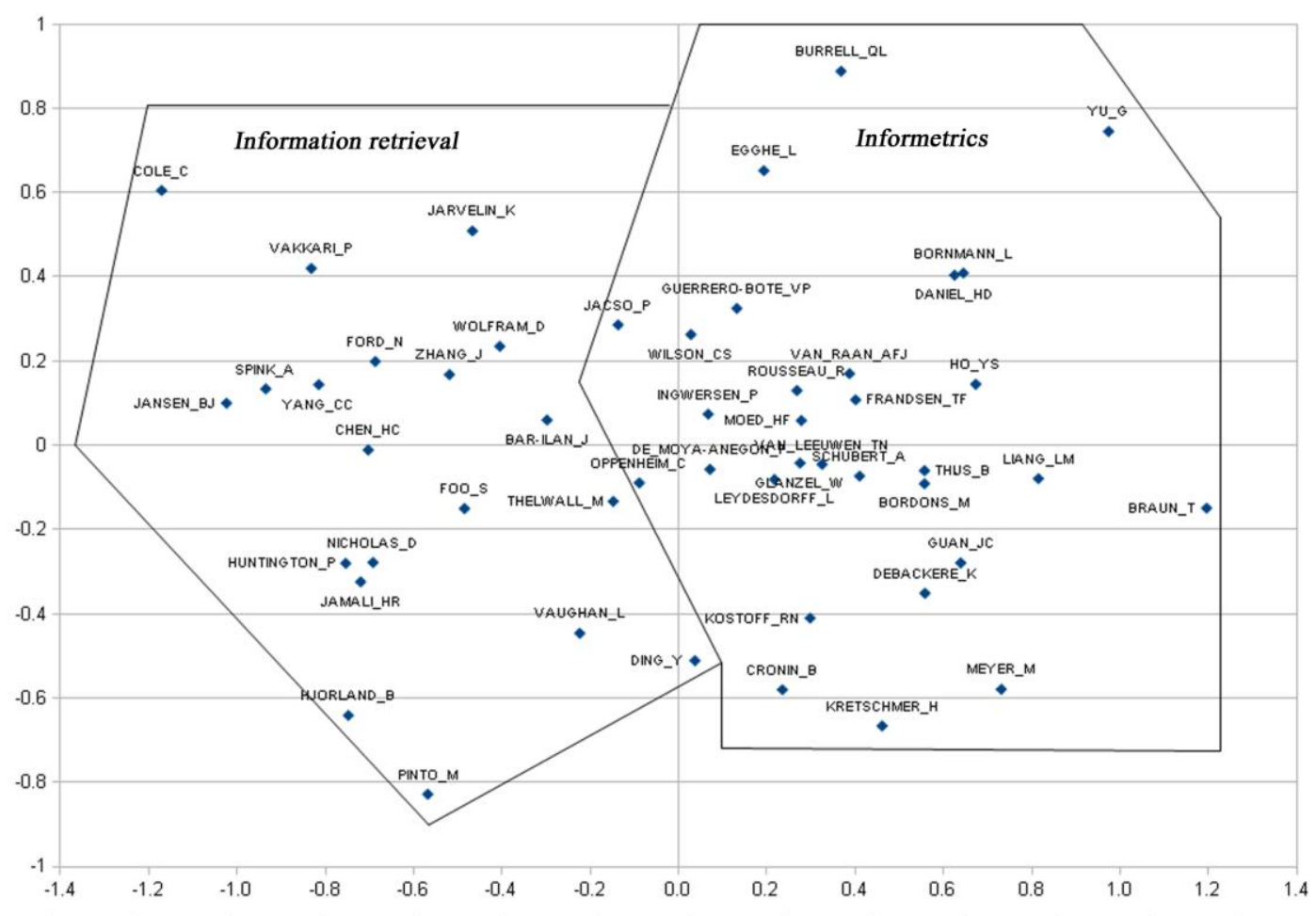

FIG. 3. Map for static author space (Normalized raw stress 0.03839). 


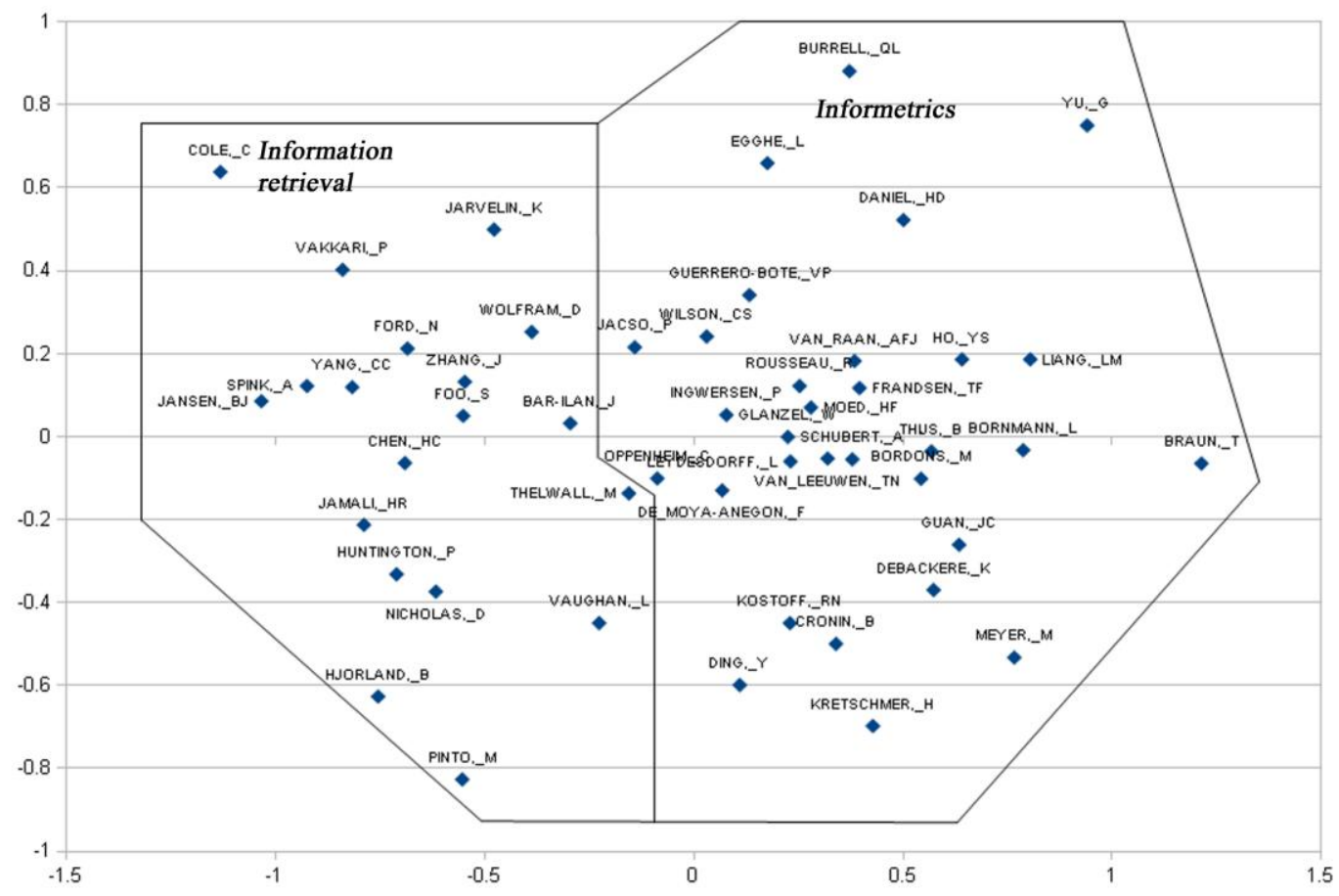

FIG. 4. Map for dynamic author space (Normalized raw stress value 0.04232).

\section{Cocitation map and LDA author map comparison}

Three distinct clusters emerge from the hierarchical cluster analysis based on cocitations (Figure 5). It can be seen from Figure 5 that the overall layout of the clusters is not as distinctive as the other content-based maps. One cluster (containing "Ho_YS", "Thelwall_M" and "Ding_Y”), dealing primarily with bibliometrics and webometrics, is situated roughly between the clusters for scientific impact evaluation and information retrieval. The clustering outcome does not provide a very coherent map of authors based on their proximities. For example, "Wilson_CS" and "Kretschmer_H" are included in the "Webometrics" group. Based on their publications used in the study they would be more appropriately categorized with the "Scientific impact evaluation" group. The lower right cluster, which contains authors who deal with the topic of information retrieval and search engine log analysis, is well defined. Some authors are positioned near the edge of the cocitation map ("Jamali_HR", "Foo_S", "Thijs_B") because they have 
received fewer citations. Their locations do not necessarily reflect their topical relatedness with the other authors in this case. The overall layout of the MDS map seems to be more central-peripheral rather than having distinctive regions. Much of the space on lower left and upper right is empty. For an exploratory purpose, one may not obtain as much information as from content-based maps. A notably low normalized raw stress value $(0.0228)$ of the cocitation map, however, indicates that the map reflects a good fit with the cocitation relationship.

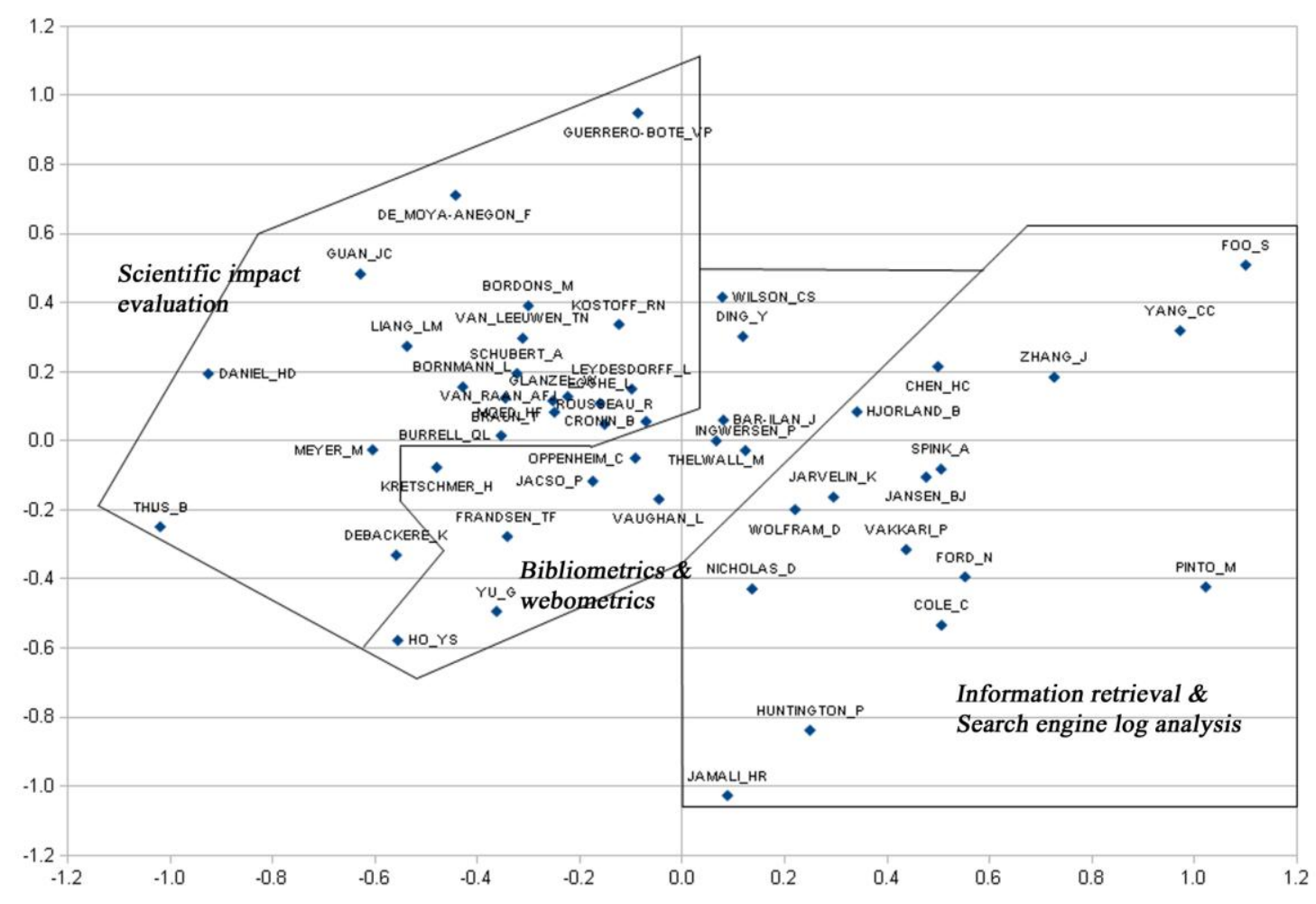

FIG. 5. Cocitation map (Normalized raw stress 0.02280).

At the four-cluster agglomeration, the LDA map (Figure 6) provides the most coherent representation of the author map in relation to the generated clusters. At the two-cluster agglomeration, the clusters are neatly divided along the vertical axis, with metrics-related research represented on the left, and Web and information retrieval-related themes on the right. Although the group membership of some individuals is 
still debatable, such as "Ingwersen_P" in the "Scientific impact evaluation" group given that he has also published in information retrieval and webometrics, the overall layout of the LDA map does provide semantically meaningful relationships.

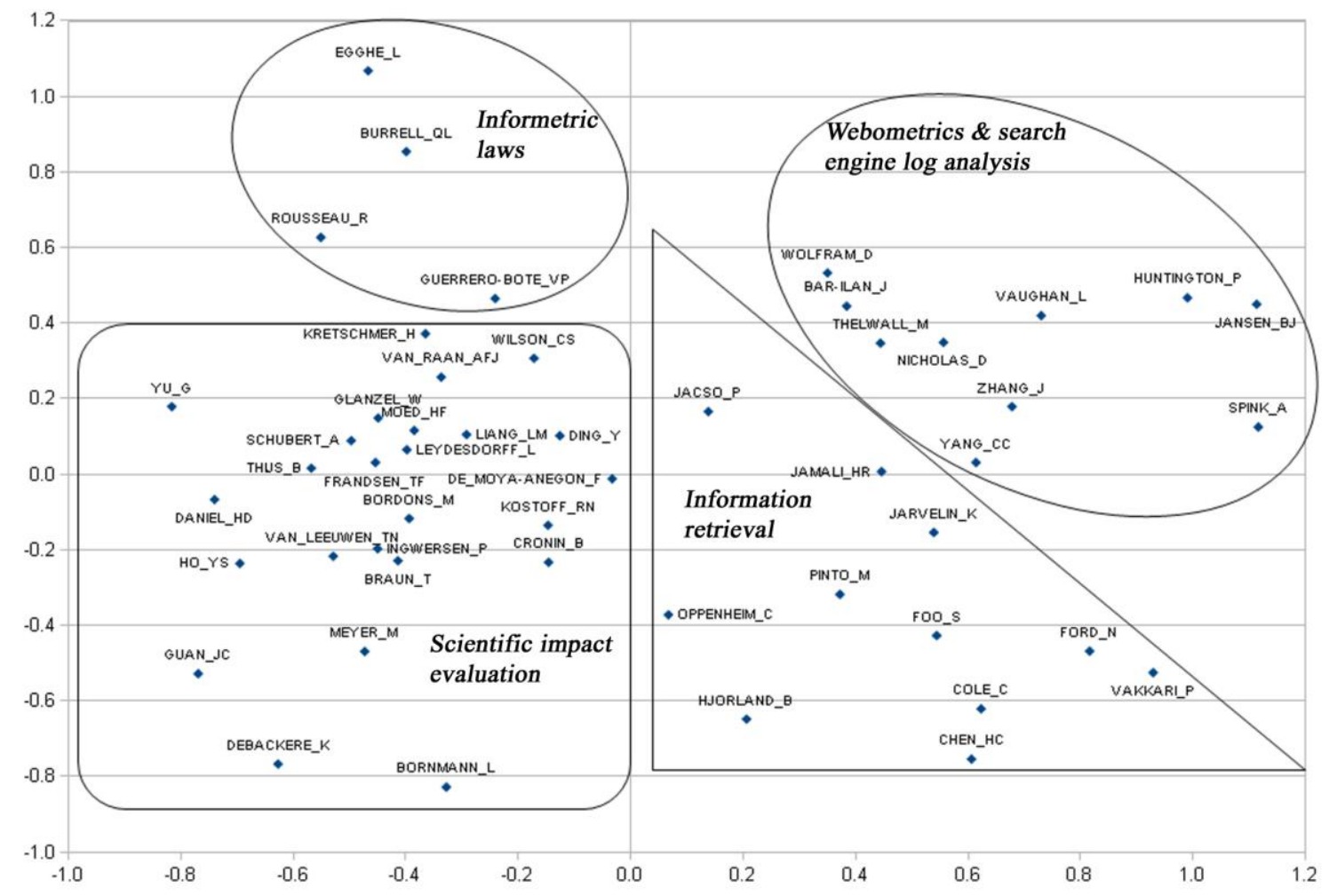

FIG. 6. Map for LDA 20 topics (Normalized raw stress 0.02856).

\section{Word-based maps and LDA map comparison}

Both word-based maps (i.e. static author map and dynamic author map) and the LDA map collect evidence of relatedness from the content of the publications. The difference between them is that the LDA map is generated based on topical similarities. When comparing the resulting maps, some notable differences can be found. First, the overall layout of the clusters in the LDA map is more distinctive than the word-based maps. The four themes are well positioned into the four quadrants of the LDA map while for the word-based maps only two themes can be distinctively identified based on the hierarchical 
clustering distance. In fact, at the two-cluster agglomeration, the two themes in the LDA map align well with those in the word-based maps. A lower normalized raw stress value for the LDA map also indicates a better fit with the data. Another notable difference is that "Yu_G" is located nearer to "Burrell_QL" than "Rousseau_R" in both word-based maps but not in the LDA map. However, checking their pairwise similarity values, "Burrell_QL" and "Rousseau_R" always have a higher similarity (0.43 for both word-based maps and 0.95 for the LDA map) than "Burrell_QL" and "Yu_G" (0.17 for both word-based maps and 0.21 for the LDA map). It is incorrectly reflected in the word-based maps due to the loss in the MDS projection. But the sharp difference of the similarity values between the two pairs in the LDA map help to retain the more accurate relationship. A further examination of their topical relatedness shows that $90 \%$ of the topical similarity between "Burrell_QL" and "Rousseau_R" is contributed by topic 5 (see appendix) which can be described as "Informetric laws", and the topical similarity between "Burrell_QL" and "Yu_G" mostly comes from topic 3 (56\%) which can be described as "Scientific impact evaluation." Our judgment agrees with this outcome after reviewing their profiles on record. Although all three authors have conducted research on scientific impact evaluation in areas such as impact factors and the h-index, "Rousseau_R" and "Burrell_QL" have more research in common by having investigated general informetric laws such as the power law model and the Lorenz/Leimkuhler function.

\section{Discussion}

Of the five author relatedness methods discussed earlier, only co-authorship provides a direct connection between authors. The other methods establish relationships based on derived similarities. These similarities are assessed based on proxies for relatedness. Cocitations are contributed by third parties. Direct citations reflect an author's assessment of relatedness to a cited author or work but are still based on perception or the subjectivity inherent in citer motivation (Bornmann \& Daniel, 2008). This is also the case for bibliographic coupling, where the strength of the relationship is assessed by the overlap of 
references selected by two authors. Co-word or topic-based studies can be argued to be the least influenced by citing behavior because they rely solely on the words developed by the authors themselves.

The use of multidimensional scaling and hierarchical cluster analysis are recognized as exploratory methods that may shed light on relationships among objects of interest that could otherwise be missed in a list of numbers. Clearly, the comparative measures used to construct the maps will influence the outcomes, so there is no single correct approach. Comparisons based on author cocitation analysis have been widely used for decades, but the word-based approaches that use similarity measures more commonly used in vector space information retrieval show some promise as well. However, as we have noted, how one includes or excludes data, such as collaborations, can affect outcomes for authors who frequently collaborate with one another.

The newly proposed content-based approaches overcome several limitations of the more traditional cocitation approach. In addition to avoiding citer subjectivity inherent in citation-based data, the links between authors will be more interpretable compared with the cocitation maps. The top terms/topics will be identifiable to help interpret the links between authors. The content-based methods do not require an author to be cited in order to be included in the map. As long as the author has some publication record, her/his relatedness with other authors can be identified. This provides the opportunity for researchers who have not been widely cited to be included in the author map. Furthermore, cocitation analysis outcomes may be affected by limited numbers of citations that do not reflect the true strength of the relationship between authors. This can be seen when comparing the cocitation outcomes with the topic-based outcomes, where several authors with low citation counts, and therefore low cocitation counts, end up at the periphery of the map. For the LDA outcome, these authors are more centrally situated among authors with similar topic areas.

The word-based and topic-based methods can be considered to be an extension of co-word analysis, where words are used to determine the relatedness of authors. The advantage of introducing the vector 
space model is that it provides more tools to formalize the relatedness measure for longer texts such as abstracts or even the full text of documents. In fact, other information retrieval models, such as language modeling (Ponte \& Croft, 1998), can be easily substituted here for the vector space model. The application of the topic model appears to be helpful in our case. Topical similarity helps to uncover some relationship otherwise hidden due to terminology mismatch, and in turn produces more sensible results, at least for library and information science. Interestingly, some other applications of topic model to author studies, such as ranking the authors (Ding, 2011a), show promise as well.

Several limitations of the research must be acknowledged. First, words are not precisely designed semantic units. Synonyms and polysemy may damage the link built based on words. Second, the LDA method does not work well for authors with limited publication records. As is the case in any other probabilistic model, an insufficient sample may lead to inferior results. However, the same would be true of cocitation analysis. Third, there are no definitive rules for identifying the number of topics to be generated in the LDA model. There will be trade-offs between an optimal level of distinctiveness and computational overhead. Next, the cluster names applied to the hierarchical cluster outcomes represent convenient labels to identify the groups generated. Although not definitive proof of outcomes, they provide evidence of potentially hidden relationships. The maps could also be interpreted without the superimposed clusters. The clusters simply provide a basis by which members of the map may be grouped. The validity of such maps has been debated for decades. In Healey, Rothman and Hoch (1986), a paradox is introduced: if a map represents a field that is already known to experts, then it is useless because it does not reveal anything new; if the map deviates from the expectation of the experts, then its outcome is questionable. This does not diminish the application of a method for exploratory purposes, particularly if topic areas or groups of authors have not been studied, or if a method has been found to be effective for known areas. Finally, one could debate whether maps based on author cocitations and content-based approaches measure the same types of relationships among authors. If the purpose of an investigation is to 
compare author relatedness based on the topics they undertake, we would propose that the content-based methods presented here provide a closer approximation toward this end.

\section{Conclusion}

In this paper we have proposed three new methods for identifying author relatedness based on the content of their work: two word-based models and one topic-based. This initial investigation, which compares prolific authors from LIS, demonstrates: (1) the potential for more topically meaningful outcomes from the new methods when compared to more traditional cocitation analysis; (2) the topic-based method using LDA for the data used in this study produces more distinctive clusters and reasonable results than the two word-based approaches. Based on the existing data, this finding cannot be generalized to other topic areas. Additional investigation is required. Advantages and disadvantages of the methods have been discussed. As an exploratory tool, author mapping doesn't currently have a gold standard evaluation measure. Subjective assessments must be made in assessing the validity of outcomes. Different methods have different perspectives and properties. The word and topic-based approaches for assessing the relatedness of research topics undertaken by authors are not intended to serve as a replacement for more established techniques like author cocitation analysis, but as additional tools for this purpose. The findings for prolific library and information science authors were particularly encouraging for the topic-based method. Future research may examine a broader range of fields.

\section{Acknowledgements}

We are grateful for the constructive comments and helpful suggestions from the anonymous reviewers.

\section{Reference}

Ahlgren, P., \& Colliander, C. (2009). Document-document similarity approaches and science mapping: Experimental comparison of five approaches. Journal of Informetrics, 3(1), 49-63. 
Ahlgren, P., Jarneving, B., \& Rousseau, R. (2003). Requirements for a cocitation similarity measure, with special reference to Pearson's correlation coefficient. Journal of the American Society for Information Science and Technology, 54(6), 550-560.

Blei, D. M., Ng, A. Y., \& Jordan, M. J. (2003). Latent Dirichlet allocation. Journal of Machine Learning Research, 3, 993-1022.

Börner, K., Chen, C., \& Boyack, K. W. (2005). Visualizing knowledge domains. Annual Review of Information Science and Technology, 37(1), 179-255.

Boyack, K. W., \& Klavans, R. (2010). Cocitation analysis, bibliographic coupling, and direct citation: Which citation approach represents the research front most accurately? Journal of the American Society for Information Science and Technology, 61(12), 2389-2404.

Bornmann, L., \& Daniel, H-D. (2008). What do citation counts measure? A review of studies on citing behavior. Journal of Documentation, 64(1), 45-80.

Braam, R. R., Moed, H. F., \& van Raan, A. F. J. (1991). Mapping of science by combined cocitation and word analysis. I. Structural Aspects. Journal of the American Society for Information Science, 42(4), 233-251.

Cao, M. D., \& Gao, X. (2005). Combining contents and citations for scientific document classification. Lecture Notes in Computer Science, 3809, 143-152.

Coulter, N., Monarch, I., \& Konda, S. (1998). Software engineering as seen through its research literature: A study in co-word analysis. Journal of the American Society for Information Science, 49(13), 1206-1223.

Deerwester, S., Dumais, S. T., Furnas, G. W., Landauer, T. K., \& Harshman, R. (1990). Indexing by latent semantic analysis. Journal of the American Society for Information Science, 41(6), 391-407.

Ding, Y. (2011a). Topic-based PageRank on author cocitation networks. Journal of the American Society for Information Science and Technology, 62(3), 449-466. 
Ding, Y. (2011b). Scientific collaboration and endorsement: Network analysis of coauthorship and citation networks. Journal of Informetrics, 5(1), 187-203.

Ding, Y., Chowdhury, G. G., \& Foo, S. (2001). Bibliometric cartography of information retrieval research by using co-word analysis. Information Processing \& Management, 37(6), 817-842.

Glänzel, W. (2001). National characteristics in international scientific co-authorship relations. Scientometrics, 51(1), 69-115.

Griffiths, T. L., \& Steyvers, M. (2004). Finding scientific topics. In Proceedings of the National Academy of Sciences of the United States of America, 101, 5228-5235.

Healey, P., Rothman, H., \& Hoch, P.K. (1986). An experiment in science mapping for research planning. Research Policy, 15(5), 233-251.

Hofmann, T. (1999). Probabilistic latent semantic indexing. In Proceedings of the $22^{\text {nd }}$ Annual International ACM SIGIR Conference on Research and Development in Information Retrieval (ACM SIGIR'1999) (pp. 50-57). Berkeley, California, USA: ACM.

Kessler, M. M. (1963). Bibliographic coupling between scientific papers. American Documentation, 14(1), $10-25$.

Law, J., \& Whittaker, J. (1992). Mapping acidification research: A test of the co-word method. Scientometrics, 23(3), 417-461.

Leydesdorff, L. (1987). Various methods for the mapping of science. Scientometrics, 11(5-6), 295-324. Leydesdorff, L. (1997). Why words and co-words cannot map the development of the sciences. Journal of the American Society for Information Science, 48(5), 418-427.

Leydesdorff, L., \& Vaughan, L. (2006). Co-occurrence matrices and their applications in information science: Extending ACA to the Web environment. Journal of the American Society for Information Science and Technology, 57(12), 1616-1628.

Liu, X., Bollen, J., Nelson, M. L., \& Van de Sompel, H. (2005). Co-authorship networks in the digital library research community. Information Processing \& Management, 41(6), 1462-1480. 
Liu, X., Yu, S., Janssens, F., Glänzel, W., Moreau, Y., \& Moor, B. D. (2010). Weighted hybrid clustering by combining text mining and bibliometrics on a large-scale journal database. Journal of the American Society for Information Science and Technology, 61(6), 1105-1119.

Ponte, J., \& Croft, W. B. (1998). A language modeling approach to information retrieval. In Proceedings of the $21^{\text {st }}$ Annual International ACM SIGIR Conference on Research and Development in Information Retrieval (ACM SIGIR'1998) (pp. 275-281). Melbourne, Australia : ACM.

Rosen-Zvi, M., Chemudugunta, C., Griffiths, T., Smyth, P., \& Steyvers, M. (2010). Learning AuthorTopic models from text corpora. ACM Transactions on Information Systems, 28(1), 1-38.

Salton, G., \& McGill, M. J. (1983). Introduction to modern information retrieval. New York: McGrawHill.

Shibata, N., Kajikawa, Y., Takeda, Y., \& Matsushima, K. (2008). Detecting emerging research fronts based on topological measures in citation networks of scientific publications. Technovation, 28(11), 758-775.

Small, H. (1973). Cocitation in the scientific literature: A new measure of the relationship between two documents. Journal of the American Society for Information Science, 24(4), 265-269.

van Eck, N. J., \& Waltman, L. (2009). How to normalize cooccurrence data? An analysis of some wellknown similarity measures. Journal of the American Society for Information Science and Technology, 60(8), 1635-1651.

Weinberg, B. H. (1974). Bibliographic coupling: A review. Information Storage and Retrieval, 10(5-6), 189-196.

White, H. D., \& Griffith, B. C. (1981). Author cocitation: A literature measure of intellectual structure. Journal of the American Society for Information Science, 32(3), 163-171.

White, H. D. (2003). Author cocitation analysis and Pearson's r. Journal of the American Society for Information Science and Technology, 54(13), 1250-1259. 
Zhao, D., \& Strotmann, A. (2008). Evolution of research activities and intellectual influences in Information Science 1996-2005: Introducing author bibliographic-coupling analysis. Journal of the American Society for Information Science and Technology, 59(13), 2070-2086.

Zhao, D., \& Strotmann, A. (2010). Mapping the highly collaborative Stem Cell research field: Adding last-author-based analysis to author cocitation analysis family. In Proceedings of the American Society for Information Science and Technology, Pittsburgh, PA, USA: ASI.

Zhao, D., \& Strotmann, A. (2011). Counting first, last, or all authors in citation analysis: A comprehensive comparison in the highly collaborative stem cell research field. Journal of the American Society for Information Science and Technology, 62(4), 654-676. 


\section{Appendix}

LDA topics and representative authors

\begin{tabular}{|c|c|c|c|c|c|c|c|}
\hline \multicolumn{2}{|c|}{ Topic 1: 0.04661} & \multicolumn{2}{|c|}{ Topic 2: $\mathbf{0 . 0 5 6 9 0}$} & \multicolumn{2}{|c|}{ Topic 3: $\mathbf{0 . 0 7 0 7 8}$} & \multicolumn{2}{|c|}{ Topic 4: $\mathbf{0 . 0 4 7 5 2}$} \\
\hline WORD & PROB & WORD & PROB & WORD & PROB & WORD & PROB \\
\hline method & 0.06436 & research & 0.06049 & citat & 0.10980 & text & 0.04916 \\
\hline cluster & 0.03788 & countri & 0.03796 & journal & 0.10174 & languag & 0.03907 \\
\hline structur & 0.03422 & patent & 0.03689 & scienc & 0.06333 & classif & 0.03144 \\
\hline map & 0.03409 & public & 0.03459 & impact & 0.05422 & word & 0.03128 \\
\hline based & 0.02608 & product & 0.03003 & author & 0.03844 & document & 0.02606 \\
\hline propos & 0.02596 & technolog & 0.02979 & public & 0.03568 & semant & 0.02366 \\
\hline similar & 0.02445 & collabor & 0.02390 & indic & 0.03552 & method & 0.02231 \\
\hline AUTHOR & PROB & AUTHOR & PROB & AUTHOR & PROB & AUTHOR & PROB \\
\hline LEYDESDORFF,_L & 0.00641 & GUAN,_JC & 0.01137 & LEYDESDORFF,_L & 0.01710 & LI,_KW & 0.00693 \\
\hline $\mathrm{AOE}, \mathrm{J}$ & 0.00504 & MEYER,_M & 0.00986 & GLANZEL,_W & 0.01177 & YANG,_CC & 0.00624 \\
\hline ZHANG,_J & 0.00357 & DEBACKERE,_K & 0.00670 & JACSO,_- & 0.00885 & $\mathrm{SEO}, \mathrm{J}$ & 0.00555 \\
\hline JARNEVING,_B & 0.00355 & GLANZEL,_W & 0.00653 & MOED,_HF & 0.00867 & THELWALL,_M & 0.00535 \\
\hline SCHNEIDER,_JW & 0.00352 & LEYDESDORFF,_L & 0.00633 & ROUSSEAU,_R & 0.00861 & CHOI,_KS & 0.00498 \\
\hline BOYACK,_KW & 0.00330 & POURIS,_A & 0.00622 & DANIEL,_HD & 0.00833 & LIU,_RL & 0.00437 \\
\hline FUKETA,_M & 0.00322 & INGWERSEN,_P & 0.00582 & TSAY,_MY & 0.00819 & LEE,_GG & 0.00412 \\
\hline \multicolumn{2}{|c|}{ Topic 5: 0.05416} & \multicolumn{2}{|c|}{ Topic 6: 0.04352} & \multicolumn{2}{|c|}{ Topic 7: 0.04825} & \multicolumn{2}{|c|}{ Topic 8: 0.04774} \\
\hline WORD & PROB & WORD & PROB & WORD & PROB & WORD & PROB \\
\hline index & 0.06295 & inform & 0.26837 & analysi & 0.08323 & knowledg & 0.09184 \\
\hline distribut & 0.03617 & make & 0.02318 & network & 0.06563 & manag & 0.04389 \\
\hline measur & 0.02721 & need & 0.02013 & social & 0.05258 & concept & 0.02963 \\
\hline paper & 0.02080 & health & 0.01760 & commun & 0.04358 & develop & 0.02483 \\
\hline number & 0.01737 & human & 0.01534 & research & 0.03647 & organ & 0.02125 \\
\hline function & 0.01499 & medic & 0.01277 & co & 0.02959 & practice & 0.02077 \\
\hline law & 0.01390 & specif & 0.01054 & field & 0.02936 & theori & 0.01954 \\
\hline
\end{tabular}




\begin{tabular}{|c|c|c|c|c|c|c|c|}
\hline$A U T H O R$ & $P R O B$ & AUTHOR & PROB & $A U T H O R$ & PROB & $A U T H O R$ & $P R O B$ \\
\hline EGGHE,_L & 0.08345 & WARNER,_J & 0.01063 & LEYDESDORFF,_L & 0.01912 & HJORLAND,_B & 0.00661 \\
\hline ROUSSEAU,_R & 0.02305 & BATH,_PA & 0.00596 & THELWALL,_M & 0.01057 & ROWLEY,_J & 0.00661 \\
\hline BURRELL,_QL & 0.01832 & WESTBROOK,_L & 0.00502 & MCCAIN,_KW & 0.00681 & DAY,_RE & 0.00531 \\
\hline GLANZEL,_W & 0.00733 & SAVOLAINEN,_R & 0.00422 & CHEN,_CM & 0.00569 & HARA,_N & 0.00480 \\
\hline SCHREIBER,_M & 0.00722 & WILLIAMS,_P & 0.00365 & WHITE,_HD & 0.00491 & CHOU,_SW & 0.00406 \\
\hline VAN_RAAN,_AFJ & 0.00649 & THELWALL,_M & 0.00359 & GLANZEL,_W & 0.00481 & CHUA,_AYK & 0.00362 \\
\hline LEYDESDORFF,_L & 0.00581 & OPPENHEIM,_C & 0.00289 & KRETSCHMER,_H & 0.00481 & JASHAPARA,_A & 0.00355 \\
\hline \multicolumn{2}{|c|}{ Topic 9: 0.0444} & \multicolumn{2}{|c|}{ Topic 10: 0.04810} & \multicolumn{2}{|c|}{ Topic 11: 0.05175} & \multicolumn{2}{|c|}{ Topic 12: 0.04906} \\
\hline WORD & PROB & WORD & PROB & WORD & PROB & WORD & PROB \\
\hline model & 0.13710 & new & 0.04420 & retriev & 0.11895 & level & 0.04039 \\
\hline base & 0.06056 & approach & 0.03513 & document & 0.07721 & number & 0.0334 \\
\hline data & 0.05257 & relat & 0.02973 & queri & 0.06940 & time & 0.02408 \\
\hline process & 0.04437 & subject & 0.02602 & term & 0.05636 & group & 0.02234 \\
\hline propos & 0.03186 & present & 0.02354 & relev & 0.05522 & statist & 0.02122 \\
\hline object & 0.02080 & suggest & 0.02113 & effect & 0.03503 & found & 0.02068 \\
\hline applic & 0.02054 & question & 0.02034 & reserv & 0.02433 & increas & 0.02014 \\
\hline AUTHOR & PROB & AUTHOR & $P R O B$ & AUTHOR & $P R O B$ & AUTHOR & PROB \\
\hline TANIGUCHI,_S & 0.00783 & THELWALL,_M & 0.01515 & JARVELIN,_K & 0.00633 & THELWALL,_M & 0.01010 \\
\hline EGGHE,_L & 0.00468 & GLANZEL,_W & 0.00524 & SAVOY,_J & 0.00573 & VAN_RAAN,_AFJ & 0.00643 \\
\hline FORD,_N & 0.00437 & JACSO,_P & 0.0048 & CRESTANI,_F & 0.00489 & SZAVAKOVATS,_E & 0.00609 \\
\hline BURRELL,_QL & 0.00404 & FORD,_N & 0.00453 & SPINK,_A & 0.00477 & GLANZEL,_W & 0.00522 \\
\hline NIEMI,_T & 0.00389 & BURRELL,_QL & 0.00394 & VECHTOMOVA,_O & 0.00465 & LEYDESDORFF,_L & 0.00452 \\
\hline THELWALL,_M & 0.00363 & MEYER,_M & 0.00358 & ZHANG,_J & 0.00423 & WOLFRAM,_D & 0.00411 \\
\hline ZHANG,_Y & 0.00306 & OPPENHEIM,_C & 0.00326 & OUNIS,_I & 0.00400 & EGGHE,_L & 0.00399 \\
\hline \multicolumn{2}{|c|}{ Topic 13: 0.04624} & \multicolumn{2}{|c|}{ Topic 14: 0.04675} & \multicolumn{2}{|c|}{ Topic 15: 0.04348} & \multicolumn{2}{|c|}{ Topic 16: 0.04912} \\
\hline WORD & PROB & WORD & \begin{tabular}{|l|} 
PROB \\
\end{tabular} & WORD & PROB & WORD & PROB \\
\hline system & 0.11421 & research & 0.07301 & internet & 0.04104 & differ & 0.08616 \\
\hline user & 0.10589 & scienc & 0.04129 & onlin & 0.03284 & result & 0.07898 \\
\hline design & 0.03744 & review & 0.03172 & technolog & 0.03022 & evalu & 0.07427 \\
\hline imag & 0.02940 & refer & 0.02612 & studi & 0.02482 & perform & 0.06775 \\
\hline content & 0.02666 & articl & 0.02470 & factor & 0.02107 & studi & 0.04713 \\
\hline interfac & 0.02215 & paper & 0.02263 & effect & 0.01963 & compar & 0.04142 \\
\hline tool & 0.01731 & literatur & 0.01955 & busi & 0.01801 & measur & 0.03779 \\
\hline AUTHOR & PROB & AUTHOR & PROB & AUTHOR & PROB & AUTHOR & PROB \\
\hline
\end{tabular}




\begin{tabular}{|c|c|c|c|c|c|c|c|}
\hline SHIRI,_A & 0.00702 & BORNMANN,_L & 0.02017 & SMITH,_AD & 0.01076 & HARTLEY,_J & 0.00498 \\
\hline JACSO,_P & 0.00477 & DANIEL,_HD & 0.01517 & LEE,_MC & 0.00547 & LEYDESDORFF,_L & 0.00435 \\
\hline CHEN,_HC & 0.00392 & THELWALL,_M & 0.00583 & GANDIA,_JL & 0.00409 & JACSO,_P & 0.00404 \\
\hline RORISSA,_A & 0.00387 & OPPENHEIM,_C & 0.00496 & FLAVIAN,_C & 0.00365 & EGGHE,_L & 0.00394 \\
\hline MARCHIONINI,_G & 0.00364 & HARTLEY,_J & 0.00396 & LEE,_MKO & 0.00365 & THELWALL,_M & 0.00387 \\
\hline FOO,_S & 0.00354 & SZAVAKOVATS,_E & 0.00386 & CASTANEDA,_JA & 0.00360 & SAVOY,_J & 0.00327 \\
\hline SHAPIRA,_B & 0.00339 & JACSO,_P & 0.00344 & CHEN,_HC & 0.00354 & GLANZEL,_W & 0.00319 \\
\hline \multicolumn{2}{|c|}{ Topic 17: 0.04594} & \multicolumn{2}{|c|}{ Topic 18: 0.05422} & \multicolumn{2}{|c|}{ Topic 19: 0.05005} & \multicolumn{2}{|c|}{ Topic 20: 0.05544} \\
\hline WORD & $P R O B$ & WORD & $P R O B$ & WORD & PROB & WORD & $P R O B$ \\
\hline articl & 0.06456 & web & 0.14779 & librari & 0.07206 & inform & 0.0903 \\
\hline databas & 0.05543 & search & 0.11097 & digit & 0.03815 & studi & 0.0458 \\
\hline research & 0.04805 & engin & 0.04466 & servic & 0.03065 & seek & 0.03793 \\
\hline univers & 0.02687 & site & 0.03651 & access & 0.03001 & behavior & 0.03632 \\
\hline rank & 0.02646 & page & 0.02661 & paper & 0.02985 & task & 0.03424 \\
\hline qualiti & 0.02076 & link & 0.02647 & resourc & 0.02978 & interact & 0.02251 \\
\hline assess & 0.01907 & result & 0.02217 & valu & 0.02849 & search & 0.02032 \\
\hline AUTHOR & PROB & AUTHOR & PROB & AUTHOR & PROB & AUTHOR & $P R O B$ \\
\hline JACSO,_P & 0.01888 & THELWALL,_M & 0.04176 & JACSO,_P & 0.01023 & SPINK,_A & 0.01373 \\
\hline THELWALL,_M & 0.01875 & JANSEN,_BJ & 0.02041 & OPPENHEIM,_C & 0.01014 & FORD,_N & 0.00985 \\
\hline BAR-ILAN,_J & 0.00868 & SPINK,_A & 0.01962 & CHOWDHURY,_GG & 0.00425 & BILAL,_D & 0.00961 \\
\hline KOUSHA,_K & 0.00712 & BAR-ILAN,_J & 0.01814 & HUNTINGTON,_P & 0.00394 & SAVOLAINEN,_R & 0.00847 \\
\hline MOED,_HF & 0.00589 & HUNTINGTON,_P & 0.01217 & NICHOLAS,_D & 0.00382 & VAKKARI,_P & 0.00804 \\
\hline KOSTOFF,_RN & 0.00521 & VAUGHAN,_L & 0.01095 & LIEW,_CL & 0.00382 & ZHANG,_Y & 0.00666 \\
\hline WILSON,_CS & 0.00511 & JACSO,_P & 0.00866 & MORRIS,_A & 0.00378 & COLE,_C & 0.00512 \\
\hline
\end{tabular}

